\title{
Lipase B from Candida antarctica - the wide applicable biocatalyst in obtaining pharmaceutical compounds
}

\author{
Corresponding author: \\ Tomasz Siódmiak \\ Department of Medicinal Chemistry, \\ Collegium Medicum in Bydgoszcz, \\ Faculty of Pharmacy, Nicolaus \\ Copernicus University in Torun, \\ Dr A. Jurasza 2, \\ 85-089 Bydgoszcz, Poland \\ e-mail: tomasz.siodmiak@cm.umk.pl
}

\begin{abstract}
Lipases are commonly applied in the pharmaceutical and chemical industry, especially in immobilized form. The use of immobilized lipases facilitates the design of reactors and control of reactions, for example, fast stopping the reaction. The immobilization procedure should increase the stability of the lipase and its activity, as well as be simple and efficient. Lipase B from Candida antarctica (CAL-B) is an enzyme from the lipase group, isolated from the Candida antarctica species. CAL-B has the highest activity in non-polar organic solvents, such as hexane and toluene, and the lowest in polar solvents, e.g. acetonitrile. Due to its hydrolytic properties, this enzyme degrades triglycerides of fatty acids to free fatty acids (FFA) and glycerol. Described lipase is often immobilized, in the aim to increase enantioselective and lipolytic activity. The kinetic and dynamic resolution with the application of lipase is one of the ways in obtaining an enantiopure form of the drugs, which usually are more effective and safer for the patient. The CAL-B could be also applied in the kinetic resolution of compounds being building blocks, derivates of drugs or conjugated forms. Furthermore, the CAL-B is used in the reactions in receiving of organic compounds, which are the natural origin, especially vegetable. Based on the presented data, it can be concluded, that CAL-B is an enzyme with a wide application in the biosynthesis of compounds with therapeutic activity. Key words: lipase B from Candida antarctica, immobilization, triglycerides, interfacial activation, kinetic resolution, transesterification, acylation
\end{abstract}

Med Res J 2019; 4 (3): 174-177

\section{Introduction}

Lipases (EC 3.1.1.3) are enzymes belonging to hydrolases (a subclass of esterases), catalyzing lipolysis, i.e. hydrolysis reaction of triacylglycerols (TAG). These molecules perform on the water-lipid interface. The widely used substrates for lipases are triglycerides (TAGs) - esters of glycerol and free fatty acids as well as phospholipids. Due to the possibility of using lipases in the synthesis or hydrolysis of many esters and amides, they are commonly applied in the pharmaceutical and chemical industry. From a medical point of view, these enzymes play a significant role in the metabolism of fatty acids in biological organisms. Most lipases are characterized by regioselectivity and stereospecificity towards specific functional groups of organic compounds, hence they are used to catalyze a number of reactions to obtain substances for use in pharmacy [1-2]. In order to improve the efficiency of biocatalysis, the process of enzyme immobilization onto supports is carried out. Immobilization gives the possibility to modify the catalytic activity of tested enzymes. The mechanism of the immobilization process is based on intermolecular interactions between the enzyme protein and the appropriate functional group of the carrier. The aim of the process is increasing the enzymatic activity and technological stability as well as facilitating the reaction control. Other benefits of the process are the easier recovery of the biocatalyst, greater thermal stability, and a wider range of activity in relation to molecular structures of substrates [3-5].

Lipase hydrolytic activity refers to the lipolysis reaction in which the triglycerides are degraded in the presence of water. It should be emphasized that the phenomenon called interfacial activation of lipases and associated with it increase of enzyme activity is widely described in the literature [6]. Moreover, in literature, there were also presented characteristic elements of the molecular structure of lipases (among others, the lid domain) allowing to control the substrate exposition 
to the active centre of the enzyme. In consequence, in a lipid-water phase, there may be a change in the conformation of the enzyme (open and close form).

\section{Lipase B from Candida antarctica (CAL-B)}

\section{Characteristic}

CAL-B is an enzyme from the lipase group, isolated from the Candida antarctica species. In terms of biochemistry, it belongs to the group of hydrolases containing the catalytic triad. Unlike other lipases, most CAL-B surfaces are naturally hydrophobic. The native form of CAL-B is active in the $\mathrm{pH}$ range from $6-10$, and its isoelectric point is 6.8. CAL-B has the highest activity in non-polar organic solvents, such as hexane and toluene, and the lowest in polar solvents, e.g. acetonitrile. It has not been proven that CAL-B lipase shows interfacial activation to a large extent, despite the high catalytic activity. This may be due to the lack of the "lid" domain, and this could influence the regulation of substrate access to the active centre. In respect of the molecular structure, the $\alpha-5$ and the $\alpha-10$ helices are the most mobile parts of the lipase structure. Molecular simulations show the movement of the $\alpha-5$ helix in the direction of the $\alpha-10$ helix, which underlines the key role of the $\alpha-5$ helix in the formation of CAL-B interaction with the hydrophobic surface. The $\alpha-5$ helix behaves like a "lid" [6]. It is believed that the open and closed structure of CAL-B is not only related to interfacial activation but also have an effect on the lipase catalytic properties. Despite the enormous progress in understanding and controlling the catalytic activity of CAL-B, the presence of the "lid" structure is discussed, and there is a lack of solid evidence regarding the interfacial activation of this enzyme. Due to its hydrolytic properties, this enzyme degrades triglycerides of fatty acids to free fatty acids (FFA) and glycerol. The reaction is reversible. In view of the high enantioselectivity, thermal stability and stability in organic solvents, this enzyme is one of the most widely used lipases in biocatalysis [7-8]. The structure of CAL-B is shown in Figure 1.

\section{Immobilization of lipase}

The use of catalysts such as lipases in many cases requires their recovery and reuse for economic and environmental reasons. The use of immobilized lipases facilitates the design of reactors and control of reactions, for example, fast stopping the reaction. Immobilization of lipases is often performed when biocatalysts are used in industrial scale. This procedure should increase the stability of the lipase and its activity, as well as be simple and efficient [11].

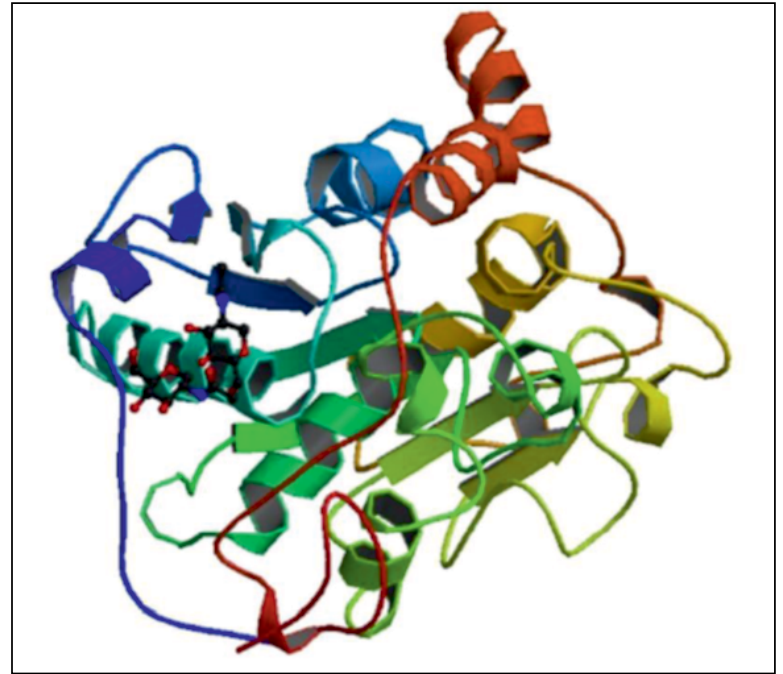

Figure 1. The crystalline structure of lipase B from Candida antarctica [9-10]

\section{Techniques of immobilization of lipases}

Many materials, including various polymer resins, cellulose polymers, silica, ceramics, carbon nanotubes, magnetic particles, and microspheres, have been tested for the immobilization of lipases.

Techniques of immobilization of lipases can be divided into four types [12]:

1) physical adsorption;

2) chemical bonding (covalent or ionic);

3) cross-linking;

4) entrapment.

Depending on the type of interaction between the carrier and the enzyme, these techniques were divided into reversible and irreversible. In the case of the irreversible methods, lipase cannot be disconnected from the support without destroying its biological activity or carrier. On the other hand, in the reversible methods, the lipase can be separated from the carrier under mild conditions. Covalent bonding, entrapment and cross-linking are the most commonly used procedures of irreversible immobilization of lipases. Physical adsorption and various non-covalent bonds such as chelation are well-known procedures for reversible immobilization.

\section{Lipase B from Candida antarctica (CAL-B) - application}

\section{Drug synthesis}

CAL-B is widely applied as a biocatalyst in biochemical and pharmaceutical reactions. This enzyme is often immobilized, in the aim of increasing its enantioselective and lipolytic activity. The immobilized form usually has 
a better ability to kinetic or dynamic kinetic resolution of a racemic mixture of enantiomers of pharmaceutical compounds. Due to enantiomers are characterized by a different pharmacodynamical profile, it is important to effectively separate each enantiomer from racemic mixtures. The kinetic and dynamic resolution is performed in order to obtain an enantiopure form of drugs, which usually are more effective and safer for the patient. An example of the application of lipases as a biocatalyst is the kinetic resolution of $(R, S)$-ibuprofen or $(R, S)$-flurbiprofen, and other drugs from the profen group. $(R)$-ibuprofen enhanced the side effects of the racemic drug on, among others, gastrointestinal and renal tract, hence it is desirable to use only the $(S)$-enantiomer. Henke et al. applied the CAL-B in the kinetic resolution of $(R, S)$-ibuprofen and the vinyl esters with high optically pure were obtained [13]. Regarding flurbiprofen, $(R)$-enantiomer is inactive on both forms of cyclooxygenase but has antinociceptive properties, and could have potential application in the treatment of prostate and colon cancer. Siódmiak et al. carried out the kinetic resolution of flurbiprofen, catalyzed by CAL-B immobilized onto the support (Novozym 435). Authors obtained the methyl ester of $(R)$-flurbiprofen with the high enantiomeric excess [14]. Another group of drugs achieved with the use of CAL-B is $\beta$-blockers, for example, $(R, S)$-propranolol. The individual enantiomers of propranolol show differences in clinical and adverse effects. Barbosa et al. achieved optically pure vinyl esters of propranolol in the reaction catalyzed by CAL-B immobilized on epoxide supports [15], whereas Sikora et al. performed the kinetic resolution of $(R, S)$-propranolol, catalyzed by native CAL-B in toluene in order to obtain (S)-propranolol acetate [16]. The CAL-B has applied also in the kinetic resolution of compounds being a building block in the synthesis of ticagrelor, one of the most important antithrombotic pharmaceutical compound. Wang et al. performed transesterification of azolide derivates (investigated for an application in ticagrelor synthesis) with methyl tert-butyl ether, catalyzed by CAL-B, in the immobilized form (Novozym 435). The reaction was enantioselective, the $E$ value was 197 [17]. Recent publications reported about CAL-B application in biosynthesis of methotrexate, anticancer and antirheumatic drug, in conjugated form. Noro et al. performed the transesterification of acylglycerols and cyclodextrins conjugated with methotrexate, catalyzed by CAL-B immobilized onto polyacrylic support, in the presence of ultrasounds [18]. CAL-B was also used in the synthesis of azelaic acid derivates. Azelaic acid is widely applied in skin diseases as inflammatory acne. The derivates of the azelaic acid are characterized by better solubility and require less dosage than acid form. The synthesis by esterification of azelaic acid with lauryl alcohol, catalyzed by CAL-B (Novozym 435) was carried out by Khairudin et al. The high conversion (> 95\%) was achieved with a small amount of enzyme [19].

\section{Synthesis of natural origins compounds}

The CAL-B is used in the reactions for receiving organic compounds, which are the natural origin, especially vegetable. One of the examples are compounds from the flavonoid group applied as a natural antioxidant. The chemical structure of flavonoids show low solubility in non-aqueous solvents, hence, this form has limited application in the biotechnological and pharmaceutical industry. The problem could be solved by acylation of flavonoids. As a result of the reaction, the acylated flavonoid can penetrate into the skin and induce the antioxidative effect. Passicos et al. developed the one-step method of acylation of routine and naringenin, with the use of immobilized CAL-B (Novozym 435) as a catalyst. The methyl palmitate was the donor of the acyl group, and the primary hydroxyl group of sugar rest of naringenin and routine was an acceptor, whereas 2-methyl-2-butanol was the solvent. For naringenin, a good result was achieved - $92 \%$ used flavonoid in the reaction was acylated [20].

The similar method in obtaining of acylated flavonoids was presented by Stevenson et al. In the case of isoquercitrin and naringenin, it achieved the significantly improve their hydrophobicity and better antioxidant effect. The acids: cinnamic, palmitate and phenylpropionic were the acyl group donor. The flavonoids were extracted from the apple and blueberry fruit. CAL-B also showed the selective acylation towards the primary hydroxyl group of the sugar portion of the flavonoid [21].

It was proven, that the application of CAL-B in receiving of an oleic ester of quercetine by acylation reaction is reasonable to greatly improve lipophilicity. The study was described by Saik et al. [22], in which the reaction catalyzed by lipase of quercetine with oleic acid as an acyl donor group was carried out.

The transesterification reaction catalyzed by CAL-B was used also in obtaining compounds present in policosanol achieved from carminic acid production. Carminic acid is a natural dye, used in the food industry. Policosanol is a mixture of long-chain alcohol, found in various plant and animal origin products, with the antiatherosclerotic properties. Ramos-Zambrano et al. performed transesterification of policosanol from waxy waste with used of CAL-B (recombinant with Aspergillus niger) immobilized on polymeric support Immobead 150 as an efficient and eco-friendly method. A different acyl donor group were applied, the most catalytically efficient was a reaction with isopropanol as a donor. The mainly obtained compound was triacontanol [23]. 


\section{Conclusions}

Lipase B from Candida antarctica is an enzyme with a wide application in the synthesis of compounds with therapeutic activity. Due to the possibility of immobilization of this enzyme onto solid supports, its multiple reuses in reactions become possible. To date, well-documented studies of the use of CAL-B in the biosynthesis indicate that with properly optimized procedures, this enzyme is one of the most appropriate and effective solutions in obtaining organic compounds for the application in medicine and pharmacy.

\section{References}

1. Stauch B, Fisher S, Cianci M. Open and closed states ofCandida antarcticalipase B: protonation and the mechanism of interfacial activation. J Lipid Res. 2015; 56(12): 2348-2358, doi: 10.1194/Jr.m063388.

2. Tan T, Lu J, Nie K, et al. Corrigendum to "Biodiesel production with immobilized lipase: A review. Biotechnol Adv. 2010; 28(6): 628-634.

3. Idris A, Bukhari A. Immobilized Candida antarctica lipase B: Hydration, stripping off and application in ring opening polyester synthesis. Biotechnol Adv. 2012; 30(3): 550-563, doi: 10.1016/j. biotechadv.2011.10.002, indexed in Pubmed: 22041165.

4. Bezerra CS, de Farias Lemos CMG, de Sousa M. Enzyme immobilization onto renewable polymeric matrixes: Past, present, and future trends. J Appl Polym Sci. 2015.

5. Mateo C, Palomo J, Fernandez-Lorente G, et al. Improvement of enzyme activity, stability and selectivity via immobilization techniques. Enzyme Microb Technol. 2007; 40(6): 1451-1463, doi: 10.1016/j. enzmictec.2007.01.018

6. Themistoklis Z, Freddolino PL, Petri T, et al. Interfacial Activation of Candida antarctica Lipase B: Combined Evidence from Experiment and Simulation. Biochemistry. 2015; 54: 5969-5979.

7. Jung S, Park S. Dual-Surface Functionalization of Metal-Organic Frameworks for Enhancing the Catalytic Activity of Candida antarctica Lipase B in Polar Organic Media. ACS Catalysis. 2016; 7(1): 438-442, doi: 10.1021/acscatal.6b03222.

8. Rabbani G, Ahmad E, Khan M, et al. Impact of structural stability of cold adapted Candida antarctica lipase $\mathrm{B}(\mathrm{CaLB})$ : in relation to $\mathrm{pH}$ chemical and thermal denaturation. RSC Advances. 2015; 5(26): 20115-20131, doi: 10.1039/c4ra17093h.

9. https://www.rcsb.org/structure/1TCA.

10. Uppenberg J, Hansen MT, Patkar S, et al. The sequence, crystal structure determination and refinement of two crystal forms of lipase B from Candida antarctica. Structure. 1994; 2(4): 293-308, indexed in Pubmed: 8087556
11. Mateo C, Palomo J, Fernandez-Lorente G, et al. Improvement of enzyme activity, stability and selectivity via immobilization techniques. Enzyme and Microbial Technology. 2007; 40(6): 1451-1463, doi: 10.1016/i.enzmictec.2007.01.018.

12. Zhao X, Qi F, Yuan C, et al. Lipase-catalyzed process for biodiesel production: Enzyme immobilization, process simulation and optimization. Renewable and Sustainable Energy Reviews. 2015; 44: 182-197, doi: 10.1016/j.rser.2014.12.021

13. Henke E, Schuster S, Yang H, et al. Lipase-Catalyzed Resolution of Ibuprofen. Monatshefte fuer Chemie/Chemical Monthly. 2000; 131(6): 633-638, doi: 10.1007/s007060070091.

14. Siódmiak T, Mangelings D, Vander Heyden $Y$, et al. High enantioselective Novozym 435-catalyzed esterification of (R,S)-flurbiprofen monitored with a chiral stationary phase. Appl Biochem Biotechnol. 2015; 175(5): 2769-2785, doi: 10.1007/s12010-014-1455-4, indexed in Pubmed: 25561056.

15. Barbosa $\mathrm{O}$, Ariza $\mathrm{C}$, Ortiz $\mathrm{C}$, et al. Kinetic resolution of (R/S)-propranolol (1-isopropylamino-3-(1-naphtoxy)-2-propanolol) catalyzed by immobilized preparations of Candida antarctica lipase B (CAL-B). N Biotechnol. 2010; 27(6): 844-850, doi: 10.1016/..nbt.2010.07.015, indexed in Pubmed: 20667519.

16. Sikora A, Tarczykowska A, Chałupka J, et al. Kinetic resolution of a b-adrenolytic drug with the use of lipases as enantioselective biocatalysts. Medical Research Journal. 2018; 3(1): 38-42, doi: 10.5603/mrj.2018.0007.

17. Wang XY, Liu JX, Tsai SW. Lipase-catalyzed hydrolytic resolution of trans-2-(3,4-difluorophenyl)cyclopropyl azolides, a key building block for Ticagrelor synthesis. Journal of the Taiwan Institute of Chemical Engineers. 2019; 97: 112-118, doi: 10.1016/j.jtice.2019.02.026

18. Noro J, Reis RL, Cavaco-Paulo A, et al. Ultrasound-assisted biosynthesis of novel methotrexate-conjugates. Ultrason Sonochem. 2018; 48: 51-56, doi: 10.1016/j.ultsonch.2018.05.017, indexed in Pubmed: 30080579

19. Khairudin N, Basri M, Fard Masoumi HR, et al. Enhancing the Bioconversion of Azelaic Acid to Its Derivatives by Response Surface Methodology. Molecules. 2018; 23(2), doi: 10.3390/molecules23020397, indexed in Pubmed: 29438284

20. Passicos E, Santarelli X, Coulon D. Regioselective acylation of flavonoids catalyzed by immobilized Candida antarctica lipase under reduced pressure. Biotechnol Lett. 2004; 26(13): 1073-1076, doi: 10.1023/b:bile.0000032967.23282.15

21. Stevenson D, Wibisono R, Jensen D, et al. Direct acylation of flavonoid glycosides with phenolic acids catalysed by Candida antarctica lipase B (Novozym 435®). Enzyme Microb Technol. 2006; 39(6): 1236-1241, doi: 10.1016/j.enzmictec.2006.03.006.

22. Saik AY, Lim YY, Stanslas J, et al. Enzymatic synthesis of quercetin oleate esters using Candida antarctica lipase B. Biotechnol Lett. 2017; 39(2): 297-304, doi: 10.1007/s10529-016-2246-5, indexed in Pubmed: 27812823.

23. Ramos-Zambrano E, Herrera-Serrano P, García-Dávila J, et al. Cochineal Waxy Residues as Source of Policosanol: Chemical Hydrolysis and Enzymatic Transesterification. Journal of Chemistry. 2019; 2019 1-10, doi: 10.1155/2019/4547378. 